\title{
Sharp bounds for the Sándor-Yang means in terms of arithmetic and contra-harmonic means
}

\author{
Hui-Zuo Xu' ${ }^{1}$ Yu-Ming Chu ${ }^{2 *}$ and Wei-Mao Qian ${ }^{3}$
}

\section{"Correspondence:}

chuyuming2005@126.com

2 Department of Mathematics,

Huzhou University, Huzhou, China

Full list of author information is

available at the end of the article

\begin{abstract}
In the article, we provide several sharp upper and lower bounds for two Sándor-Yang means in terms of combinations of arithmetic and contra-harmonic means.
\end{abstract}

MSC: Primary 26E60; secondary 26D07; 26D99

Keywords: Schwab-Borchardt mean; Sándor-Yang mean; Arithmetic mean; Contra-harmonic mean; Quadratic mean

\section{Preliminaries}

Let $a, b>0$ with $a \neq b$. Then the arithmetic mean $A(a, b)$ [1-4], the quadratic mean $Q(a, b)$ [5], the contra-harmonic mean $C(a, b)$ [6-9], the Neuman-Sándor mean NS(a,b) [10-12], the second Seiffert mean $T(a, b)[13,14]$, and the Schwab-Borchardt mean $S B(a, b)[15$, 16] of $a$ and $b$ are defined by

$$
\begin{aligned}
& A(a, b)=\frac{a+b}{2}, \quad Q(a, b)=\sqrt{\frac{a^{2}+b^{2}}{2}}, \quad C(a, b)=\frac{a^{2}+b^{2}}{a+b}, \\
& N S(a, b)=\frac{a-b}{2 \sinh ^{-1}\left(\frac{a-b}{a+b}\right)}, \\
& T(a, b)=\frac{a-b}{2 \arctan \left(\frac{a-b}{a+b}\right)}, \\
& S B(a, b)= \begin{cases}\frac{\sqrt{b^{2}-a^{2}}}{\arccos (a / b)}, & a<b, \\
\frac{\sqrt{a^{2}-b^{2}}}{\cosh ^{-1}(a / b)}, & a>b,\end{cases}
\end{aligned}
$$

respectively, where $\sinh ^{-1}(x)=\log \left(x+\sqrt{x^{2}+1}\right)$ and $\cosh ^{-1}(x)=\log \left(x+\sqrt{x^{2}-1}\right)$ are respectively the inverse hyperbolic sine and cosine functions. The Schwab-Borchardt mean $S B(a, b)$ is strictly increasing, non-symmetric and homogeneous of degree one with respect to its variables. It can be expressed by the degenerated completely symmetric elliptic integral of the first kind [17]. Recently, the Schwab-Borchardt mean has attracted the attention of many researchers. In particular, many remarkable inequalities for the SchwabBorchardt mean and its generated means can be found in the literature [18-38]. 
Let $X(a, b)$ and $Y(a, b)$ denote symmetric bivariate means of $a$ and $b$. Then Yang [39] introduced the Sándor-Yang mean

$$
R_{X Y}(a, b)=Y(a, b) e^{\frac{X(a, b)}{S B[X(a, b), Y(a, b)]}-1}
$$

and presented the explicit formulas for $R_{Q A}(a, b)$ and $R_{A Q}(a, b)$ as follows:

$$
\begin{aligned}
& R_{Q A}(a, b)=A(a, b) e^{\frac{Q(a, b)}{N S(a, b)}-1} \\
& R_{A Q}(a, b)=Q(a, b) e^{\frac{A(a, b)}{T(a, b)}-1} .
\end{aligned}
$$

Very recently, the bounds involving the Sándor-Yang means have been the subject of intensive research. Numerous interesting results and inequalities for $R_{Q A}(a, b)$ and $R_{A Q}(a, b)$ can be found in the literature [40-42].

Neuman [43] established the inequality

$$
R_{A Q}(a, b)<R_{Q A}(a, b)
$$

for $a, b>0$ with $a \neq b$.

In [44], Xu proved that the double inequalities

$$
\begin{aligned}
& \alpha_{1} C(a, b)+\left(1-\alpha_{1}\right) A(a, b)<R_{Q A}(a, b)<\beta_{1} C(a, b)+\left(1-\beta_{1}\right) A(a, b), \\
& \alpha_{2} C(a, b)+\left(1-\alpha_{2}\right) A(a, b)<R_{A Q}(a, b)<\beta_{2} C(a, b)+\left(1-\beta_{2}\right) A(a, b)
\end{aligned}
$$

hold for all $a, b>0$ with $a \neq b$ if and only if $\alpha_{1} \leq(1+\sqrt{2})^{\sqrt{2}} / e-1=0.2794 \ldots, \beta_{1} \geq 1 / 3$, $\alpha_{2} \leq \sqrt{2} e^{\pi / 4-1}-1=0.1410 \ldots$ and $\beta_{2} \geq 1 / 6$.

From (1.6) and (1.7), together the well-known inequalities

$$
C(a, b)>Q(a, b)>A(a, b), \quad Q(a, b)>\frac{1}{3} C(a, b)+\frac{2}{3} A(a, b),
$$

we clearly see that

$$
A(a, b)<R_{A Q}(a, b)<R_{Q A}(a, b)<Q(a, b)<C(a, b)
$$

for all $a, b>0$ with $a \neq b$.

The main purpose of this paper is to find the best possible parameters $\alpha_{i}, \beta_{i} \in(0,1)$ $(i=1,2,3,4)$ such that the double inequalities

$$
\begin{aligned}
& C^{\alpha_{1}}(a, b) A^{1-\alpha_{1}}(a, b)<R_{Q A}(a, b)<C^{\beta_{1}}(a, b) A^{1-\beta_{1}}(a, b), \\
& C^{\alpha_{2}}(a, b) A^{1-\alpha_{2}}(a, b)<R_{A Q}(a, b)<C^{\beta_{2}}(a, b) A^{1-\beta_{2}}(a, b), \\
& \alpha_{3}\left[\frac{1}{3} C(a, b)+\frac{2}{3} A(a, b)\right]+\left(1-\alpha_{3}\right) C^{1 / 3}(a, b) A^{2 / 3}(a, b) \\
& <R_{Q A}(a, b)<\beta_{3}\left[\frac{1}{3} C(a, b)+\frac{2}{3} A(a, b)\right]+\left(1-\beta_{3}\right) C^{1 / 3}(a, b) A^{2 / 3}(a, b),
\end{aligned}
$$




$$
\begin{aligned}
\alpha_{4} & {\left[\frac{1}{6} C(a, b)+\frac{5}{6} A(a, b)\right]+\left(1-\alpha_{4}\right) C^{1 / 6}(a, b) A^{5 / 6}(a, b) } \\
<R_{A Q}(a, b) & <\beta_{4}\left[\frac{1}{6} C(a, b)+\frac{5}{6} A(a, b)\right]+\left(1-\beta_{4}\right) C^{1 / 6}(a, b) A^{5 / 6}(a, b)
\end{aligned}
$$

hold for all $a, b>0$ with $a \neq b$.

\section{Lemmas}

In order to prove our main results, we need several lemmas, which we present in this section.

Lemma 2.1 (see [45]) Let $a, b \in \mathbb{R}$ with $a<b, f, g:[a, b] \mapsto \mathbb{R}$ be continuous on $[a, b]$ and differentiable on $(a, b)$, and $g^{\prime}(x) \neq 0$ on $(a, b)$. If $f^{\prime}(x) / g^{\prime}(x)$ is increasing (decreasing) on $(a, b)$, then so are the functions

$$
\frac{f(x)-f(a)}{g(x)-g(a)}, \quad \frac{f(x)-f(b)}{g(x)-g(b)} .
$$

If $f^{\prime}(x) / g^{\prime}(x)$ is strictly monotone, then the monotonicity in the conclusion is also strict.

Lemma 2.2 (see [46]) Let $A(t)=\sum_{k=0}^{\infty} a_{k} t^{k}$ and $B(t)=\sum_{k=0}^{\infty} b_{k} t^{k}$ be two real power series converging on $(-r, r)(r>0)$ with $b_{k}>0$ for all $k$. If the non-constant sequence $\left\{a_{k} / b_{k}\right\}_{k=0}^{\infty}$ is increasing (decreasing) for all $k$, then the function $t \mapsto A(t) / B(t)$ is strictly increasing (decreasing) on $(0, r)$.

Lemma 2.3 The function

$$
\phi(x)=\frac{x \operatorname{coth}(x)-1}{2 \log [\cosh (x)]}
$$

is strictly increasing from $(0, \log (1+\sqrt{2})$ onto $(1 / 3,[\sqrt{2} \log (1+\sqrt{2})-1] / \log 2)$.

Proof Let $\phi_{1}(x)=x \operatorname{coth}(x)-1, \phi_{2}(x)=2 \log [\cosh (x)]$. Then elaborate computations lead to

$$
\begin{aligned}
\phi(x)= & \frac{\phi_{1}(x)}{\phi_{2}(x)}=\frac{\phi_{1}(x)-\phi_{1}\left(0^{+}\right)}{\phi_{2}(x)-\phi_{2}(0)}, \\
\frac{\phi_{1}^{\prime}(x)}{\phi_{2}^{\prime}(x)}= & \frac{\sinh (x) \cosh ^{2}(x)-x \cosh (x)}{2 \sinh ^{3}(x)} \\
& =\frac{\sinh (3 x)+\sinh (x)-4 x \cosh (x)}{2 \sinh (3 x)-6 \sinh (x)}=\frac{\sum_{n=0}^{\infty} \frac{3^{2 n+1}-8 n-3}{(2 n+1) !} x^{2 n+1}}{\sum_{n=0}^{\infty} \frac{6\left(3^{2 n}-1\right)}{(2 n+1) !} x^{2 n+1}} \\
& =\frac{\sum_{n=1}^{\infty} \frac{3^{2 n+1}-8 n-3}{(2 n+1) !} x^{2 n+1}}{\sum_{n=1}^{\infty} \frac{6\left(3^{2 n}-1\right)}{(2 n+1) !} x^{2 n+1}}=\frac{\sum_{n=0}^{\infty} \frac{3^{2 n+3}-8 n-11}{(2 n+3) !} x^{2 n+3}}{\sum_{n=0}^{\infty} \frac{6\left(3^{2 n+2}-1\right)}{(2 n+3) !} x^{2 n+3}} .
\end{aligned}
$$

Let

$$
a_{n}=\frac{3^{2 n+3}-8 n-11}{(2 n+3) !}, \quad b_{n}=\frac{6\left(3^{2 n+2}-1\right)}{(2 n+3) !} .
$$


Then

$$
b_{n}>0
$$

and

$$
\frac{a_{n+1}}{b_{n+1}}-\frac{a_{n}}{b_{n}}=\frac{4\left[(72 n+63) 3^{2 n}+1\right]}{3\left(3^{2 n+4}-1\right)\left(3^{2 n+2}-1\right)}>0
$$

for all $n \geq 0$.

It follows from Lemma 2.2 and $(2.2)-(2.5)$ that $\phi_{1}^{\prime}(x) / \phi_{2}^{\prime}(x)$ is strictly increasing on $(0, \log (1+\sqrt{2}))$.

Note that

$$
\phi\left(0^{+}\right)=\frac{a_{0}}{b_{0}}=\frac{1}{3}, \quad \phi(\log (1+\sqrt{2}))=\frac{\sqrt{2} \log (1+\sqrt{2})-1}{\log 2}=0.3555 \ldots
$$

Therefore, Lemma 2.3 follows from Lemma 2.1, (2.1), and (2.6) together with the monotonicity of $\phi_{1}^{\prime}(x) / \phi_{2}^{\prime}(x)$.

Lemma 2.4 The function

$$
\varphi(x)=\frac{\log \sec (x)+x \cot (x)-1}{2 \log \sec (x)}
$$

is strictly increasing from $(0, \pi / 4)$ onto $(1 / 6,1 / 2-(4-\pi)(4 \log 2))$.

Proof Let $\varphi_{1}(x)=\log \sec (x)+x \cot (x)-1, \varphi_{2}(x)=2 \log [\sec (x)], \varphi_{3}(x)=\sin (x)-x \cos (x)$, and $\varphi_{4}(x)=2 \sin ^{3}(x)$. Then elaborate computations lead to

$$
\begin{aligned}
& \varphi(x)=\frac{\varphi_{1}(x)}{\varphi_{2}(x)}=\frac{\varphi_{1}(x)-\varphi_{1}\left(0^{+}\right)}{\varphi_{2}(x)-\varphi_{2}(0)} \\
& \frac{\varphi_{1}^{\prime}(x)}{\varphi_{2}^{\prime}(x)}=\frac{\varphi_{3}(x)}{\varphi_{4}(x)}=\frac{\varphi_{3}(x)-\varphi_{3}(0)}{\varphi_{4}(x)-\varphi_{4}(0)}
\end{aligned}
$$

and

$$
\frac{\varphi_{3}^{\prime}(x)}{\varphi_{4}^{\prime}(x)}=\frac{x}{3 \sin (2 x)}=\frac{1}{6} \times \frac{1}{\sin (2 x) /(2 x)} .
$$

It is well known that the function $x \rightarrow \sin (x) / x$ is strictly decreasing on $(0, \pi / 2)$, hence equation (2.9) leads to the conclusion that the function $\varphi_{3}^{\prime}(x) / \varphi_{4}^{\prime}(x)$ is strictly increasing on $(0, \pi / 4)$.

Note that

$$
\begin{aligned}
& \varphi\left(0^{+}\right)=\lim _{x \rightarrow 0^{+}} \frac{\varphi_{3}^{\prime}(x)}{\varphi_{4}^{\prime}(x)}=\frac{1}{6}, \\
& \varphi\left(\frac{\pi}{4}\right)=\frac{1}{2}-\frac{4-\pi}{4 \log 2}=0.1903 \ldots .
\end{aligned}
$$


Therefore, Lemma 2.4 follows from Lemma 2.1 and (2.7)-(2.9) together with the monotonicity of $\varphi_{3}^{\prime}(x) / \varphi_{4}^{\prime}(x)$.

Lemma 2.5 Let $p \in(0,1)$ and

$$
f(x)=3 p^{2} x^{10}+14 p(1-p) x^{6}+18 p^{2} x^{4}-9(1-p)^{2} x^{2}-2 p(1-p)
$$

Then the following statements are true:

(1) If $p=3 / 10$, then $f(x)>0$ for all $x \in(1, \sqrt[6]{2})$;

(2) If $p=3\left[(1+\sqrt{2})^{\sqrt{2}} / e-\sqrt[3]{2}\right] /(4-3 \sqrt[3]{2})=0.2663 \ldots$, then there exists

$\lambda_{0}(=1.0808 \ldots) \in(1, \sqrt[6]{2})$ such that $f(x)<0$ for $x \in\left(1, \lambda_{0}\right)$ and $f(x)>0$ for

$x \in\left(\lambda_{0}, \sqrt[6]{2}\right)$.

Proof Part (1) follows easily from

$$
f(x)=\frac{3}{100}\left(x^{2}-1\right)\left(9 x^{8}+9 x^{6}+107 x^{4}+161 x^{2}+14\right)>0
$$

for all $x \in(1, \sqrt[6]{2})$ if $p=3 / 10$.

For part (2), if $p=3\left[(1+\sqrt{2})^{\sqrt{2}} / e-\sqrt[3]{2}\right] /(4-3 \sqrt[3]{2})$, then numerical computations lead to

$$
\begin{aligned}
& 20 p-3=2.3273 \cdots>0 \\
& f(1)=3(10 p-3)=-1.008 \cdots<0 \\
& f(\sqrt[6]{2})=1.6809 \cdots>0 \\
& f^{\prime}(x)=30 p^{2} x^{9}+84 p(1-p) x^{5}+72 p^{2} x^{3}-18(1-p)^{2} x
\end{aligned}
$$

It follows from (2.11) and (2.14) that

$$
f^{\prime}(x)>\left[30 p^{2}+84 p(1-p)+72 p^{2}-18(1-p)^{2}\right] x=6(20 p-3) x>0
$$

for all $x \in(1, \sqrt[6]{2})$.

Therefore, part (2) follows easily from (2.12), (2.13), (2.15), and the numerical results $f(1.0808)<0$ and $f(1.0809)>0$.

Lemma 2.6 Let $p \in(0,1)$ and

$$
g(x)=3 p^{2} x^{11}+56 p(1-p) x^{6}+75 p^{2} x^{5}-72(1-p)^{2} x-50 p(1-p) .
$$

\section{Then the following statements are true:}

(1) If $p=12 / 25$, then $g(x)>0$ for all $x \in(1, \sqrt[6]{2})$;

(2) If $p=6\left[\sqrt{2} e^{\pi / 4-1}-\sqrt[6]{2}\right] /(7-6 \sqrt[6]{2})=0.4210 \ldots$, then there exists $\mu_{0}(=1.0577 \ldots) \in(1, \sqrt[6]{2})$ such that $g(x)<0$ for $x \in\left(1, \mu_{0}\right)$ and $g(x)>0$ for $x \in\left(\mu_{0}, \sqrt[6]{2}\right)$. 
Proof Part (1) follows easily from

$$
\begin{aligned}
g(x)= & \frac{24}{625}(x-1)\left(18 x^{10}+18 x^{9}+18 x^{8}+18 x^{7}+18 x^{6}+382 x^{5}+832 x^{4}\right. \\
& \left.+832 x^{3}+832 x^{2}+832 x+325\right)>0
\end{aligned}
$$

for all $x \in(1, \sqrt[6]{2})$ if $p=12 / 25$.

For part (2), if $p=6\left[\sqrt{2} e^{\pi / 4-1}-\sqrt[6]{2}\right] /(7-6 \sqrt[6]{2})=0.4210 \ldots$, then numerical computations lead to

$$
\begin{aligned}
& 20 p-3=5.4217 \cdots>0, \\
& g(1)=6(25 p-12)=-8.8367 \cdots<0, \\
& g(\sqrt[6]{2})=13.6200 \cdots>0, \\
& g^{\prime}(x)=3\left[11 p^{2} x^{10}+112 p(1-p) x^{5}+125 p^{2} x^{4}-24(1-p)^{2}\right] .
\end{aligned}
$$

It follows from (2.16) and (2.19) that

$$
\begin{aligned}
g^{\prime}(x) & >11 p^{2}+112 p(1-p)+125 p^{2}-24(1-p)^{2} \\
& =24(20 p-3)>0
\end{aligned}
$$

for $x \in(1, \sqrt[6]{2})$.

Therefore, part (2) follows easily from (2.17), (2.18), and (2.20) together with the numerical results $g(1.0577)<0$ and $g(1.0578)>0$.

\section{Main results}

We are now in a position to state and prove our main results.

Theorem 3.1 The double inequality

$$
C^{\alpha_{1}}(a, b) A^{1-\alpha_{1}}(a, b)<R_{Q A}(a, b)<C^{\beta_{1}}(a, b) A^{1-\beta_{1}}(a, b)
$$

holds for all $a, b>0$ with $a \neq b$ if and only if $\alpha_{1} \leq 1 / 3$ and $\beta_{1} \geq[\sqrt{2} \log (1+\sqrt{2})-1] / \log 2$.

Proof Clearly, inequality (3.1) can be rewritten as

$$
\left[\frac{C(a, b)}{A(a, b)}\right]^{\alpha_{1}}<\frac{R_{Q A}(a, b)}{A(a, b)}<\left[\frac{C(a, b)}{A(a, b)}\right]^{\beta_{1}}
$$

Since $A(a, b), R_{Q A}(a, b)$, and $C(a, b)$ are symmetric and homogenous of degree one, we assume that $a>b>0$. Let $v=(a-b) /(a+b) \in(0,1)$. Then from (1.1), (1.2), and (1.4) we know that inequality (3.2) is equivalent to

$$
\alpha_{1}<\frac{\left[\sqrt{1+v^{2}} \sinh ^{-1}(v)\right] / v-1}{\log \left(1+v^{2}\right)}<\beta_{1} .
$$


Let $x=\sinh ^{-1}(v)$. Then $x \in(0, \log (1+\sqrt{2}))$ and

$$
\frac{\left[\sqrt{1+v^{2}} \sinh ^{-1}(v)\right] / v-1}{\log \left(1+v^{2}\right)}=\frac{x \operatorname{coth}(x)-1}{2 \log [\cosh (x)]}:=\phi(x) .
$$

Therefore, inequality (3.1) holds for all $a, b>0$ with $a \neq b$ if and only if $\alpha_{1} \leq 1 / 3$ and $\beta_{1} \geq[\sqrt{2} \log (1+\sqrt{2})-1] / \log 2$ follows from (3.2)-(3.4) and Lemma 2.3.

Theorem 3.2 The double inequality

$$
C^{\alpha_{2}}(a, b) A^{1-\alpha_{2}}(a, b)<R_{A Q}(a, b)<C^{\beta_{2}}(a, b) A^{1-\beta_{2}}(a, b)
$$

holds for all $a, b>0$ with $a \neq b$ if and only if $\alpha_{2} \leq 1 / 6$ and $\beta_{2} \geq 1 / 2-(4-\pi) /(4 \log 2)=$ $0.1903 \ldots$. .

Proof Clearly, inequality (3.5) can be rewritten as

$$
\left[\frac{C(a, b)}{A(a, b)}\right]^{\alpha_{2}}<\frac{R_{A Q}(a, b)}{A(a, b)}<\left[\frac{C(a, b)}{A(a, b)}\right]^{\beta_{2}} .
$$

Since $A(a, b), R_{A Q}(a, b)$, and $C(a, b)$ are symmetric and homogenous of degree one, we assume that $a>b>0$. Let $v=(a-b) /(a+b) \in(0,1)$. Then from (1.1), (1.3), and (1.5) we see that inequality (3.6) is equivalent to

$$
\alpha_{2}<\frac{\log \sqrt{1+v^{2}}+[\arctan (v)] / v-1}{\log \left(1+v^{2}\right)}<\beta_{2} .
$$

Let $x=\arctan (v)$. Then $x \in(0, \pi / 4)$ and

$$
\begin{aligned}
& \frac{\log \sqrt{1+v^{2}}+[\arctan (v)] / v-1}{\log \left(1+v^{2}\right)} \\
& =\frac{\log \sec (x)+x \cot (x)-1}{2 \log \sec (x)}:=\varphi(x) .
\end{aligned}
$$

Therefore, inequality (3.5) holds for all $a, b>0$ with $a \neq b$ if and only if $\alpha_{2} \leq 1 / 6$ and $\beta_{2} \geq 1 / 2-(4-\pi) /(4 \log 2)=0.1903 \ldots$ follows from (3.6)-(3.8) and Lemma 2.4 .

Theorem 3.3 The double inequality

$$
\begin{aligned}
& \alpha_{3} {\left[\frac{1}{3} C(a, b)+\frac{2}{3} A(a, b)\right]+\left(1-\alpha_{3}\right) C^{1 / 3}(a, b) A^{2 / 3}(a, b) } \\
&<R_{Q A}(a, b)<\beta_{3}\left[\frac{1}{3} C(a, b)+\frac{2}{3} A(a, b)\right]+\left(1-\beta_{3}\right) C^{1 / 3}(a, b) A^{2 / 3}(a, b)
\end{aligned}
$$

holds for all $a, b>0$ with $a \neq b$ if and only if $\alpha_{3} \leq 3\left[(1+\sqrt{2})^{\sqrt{2}} / e-\sqrt[3]{2}\right] /(4-3 \sqrt[3]{2})=$ $0.2663 \ldots$ and $\beta_{3} \geq 3 / 10$. 
Proof Since $R_{Q A}(a, b), A(a, b)$, and $C(a, b)$ are symmetric and homogenous of degree one, without loss generality, we assume that $a>b>0$. Let $v=(a-b) /(a+b), x=\sqrt[6]{1+v^{2}}$, and $p \in(0,1)$. Then $v \in(0,1), x \in(1, \sqrt[6]{2})$, and (1.1), (1.2), and (1.4) lead to

$$
\begin{aligned}
& \log \frac{R_{Q A}(a, b)}{p\left[\frac{1}{3} C(a, b)+\frac{2}{3} A(a, b)\right]+(1-p) C^{1 / 3}(a, b) A^{2 / 3}(a, b)} \\
& \quad=\frac{\sqrt{1+v^{2}} \sinh ^{-1}(v)}{v}-\log \left[p\left(\frac{1}{3} v^{2}+1\right)+(1-p) \sqrt[3]{1+v^{2}}\right]-1 \\
& \quad=\frac{x^{3} \sinh ^{-1}\left(\sqrt{x^{6}-1}\right)}{\sqrt{x^{6}-1}}-\log \left[p\left(\frac{1}{3} x^{6}+\frac{2}{3}\right)+(1-p) x^{2}\right]-1 .
\end{aligned}
$$

Let

$$
F(x)=\frac{x^{3} \sinh ^{-1}\left(\sqrt{x^{6}-1}\right)}{\sqrt{x^{6}-1}}-\log \left[p\left(\frac{1}{3} x^{6}+\frac{2}{3}\right)+(1-p) x^{2}\right]-1 .
$$

Then simple computations lead to

$$
\begin{aligned}
& F\left(1^{+}\right)=0, \quad F(\sqrt[6]{2})=\sqrt{2} \log (1+\sqrt{2})-\log \left[\frac{4}{3} p+\sqrt[3]{2}(1-p)\right]-1, \\
& F^{\prime}(x)=\frac{3 x^{2}}{\left(x^{6}-1\right)^{3 / 2}} F_{1}(x)
\end{aligned}
$$

where

$$
\begin{aligned}
& F_{1}(x)=\frac{\sqrt{x^{6}-1}\left[-p x^{10}+(1-p) x^{6}+4 p x^{4}+2(1-p)\right]}{x\left[p\left(x^{6}+2\right)+3(1-p) x^{2}\right]}-\sinh ^{-1}\left(\sqrt{x^{6}-1}\right), \\
& F_{1}(1)=0, \quad F_{1}(\sqrt[6]{2})=\frac{(\sqrt[6]{2048}-2 \sqrt{2}) p-\sqrt[6]{2048}}{3 \sqrt[3]{2} p-3 \sqrt[3]{2}-4 p}-\log (1+\sqrt{2}) \\
& F_{1}^{\prime}(x)=-\frac{2\left(x^{6}-1\right)^{3 / 2}}{x^{2}\left[p\left(x^{6}+2\right)+3(1-p) x^{2}\right]^{2}} f(x)
\end{aligned}
$$

where $f(x)$ is defined as in Lemma 2.5.

We divide the proof into four cases.

Case $1 p=3 / 10$. Then it follows from (3.9)-(3.14) and Lemma 2.5(1) that

$$
R_{Q A}(a, b)<\frac{3}{10}\left[\frac{1}{3} C(a, b)+\frac{2}{3} A(a, b)\right]+\frac{7}{10} C^{1 / 3}(a, b) A^{2 / 3}(a, b) .
$$

Case $20<p<3 / 10$. Let $v>0$ and $v \rightarrow 0^{+}$. Then power series expansion leads to

$$
\begin{aligned}
& \frac{\sqrt{1+v^{2}} \sinh ^{-1}(v)}{v}-\log \left[p\left(\frac{1}{3} v^{2}+1\right)+(1-p) \sqrt[3]{1+v^{2}}\right]-1 \\
& =\left(\frac{1}{30}-\frac{1}{9} p\right) v^{4}+O\left(v^{6}\right)
\end{aligned}
$$


Equations (3.9), (3.10), and (3.15) lead to the conclusion that there exists $0<\delta_{1}<1$ such that

$$
R_{Q A}(a, b)>p\left[\frac{1}{3} C(a, b)+\frac{2}{3} A(a, b)\right]+(1-p) C^{1 / 3}(a, b) A^{2 / 3}(a, b)
$$

for all $a>b>0$ with $(a-b) /(a+b) \in\left(0, \delta_{1}\right)$.

Case $3 p=3\left[(1+\sqrt{2})^{\sqrt{2}} / e-\sqrt[3]{2}\right] /(4-3 \sqrt[3]{2})$. Then (3.13) leads to

$$
F_{1}(\sqrt[6]{2})=-0.0039 \cdots<0
$$

Let $\lambda_{0}=1.0808 \ldots$ be the number given in Lemma 2.5(2). Then we divide the discussion into two subcases.

Subcase $1 x \in\left(1, \lambda_{0}\right]$. Then $F_{1}(x)>0$ for $x \in\left(1, \lambda_{0}\right]$ follows easily from (3.13) and (3.14) together with Lemma 2.5(2).

Subcase $2 x \in\left(\lambda_{0}, \sqrt[6]{2}\right)$. Then Lemma 2.5(2) and (3.14) lead to the conclusion that $F_{1}(x)$ is strictly decreasing on the interval $\left[\lambda_{0}, \sqrt[6]{2}\right)$. Then, from (3.16) and Subcase 1 , we know that there exists $\lambda_{1} \in\left(\lambda_{0}, \sqrt[6]{2}\right)$ such that $F_{1}(x)>0$ for $x \in\left[\lambda_{0}, \lambda_{1}\right)$ and $F_{1}(x)<0$ for $x \in\left(\lambda_{1}, \sqrt[6]{2}\right)$.

It follows from Subcases 1 and 2 together with (3.12) that $F(x)$ is strictly increasing on $\left(1, \lambda_{1}\right]$ and strictly decreasing on $\left[\lambda_{1}, \sqrt[6]{2}\right)$. Therefore,

$$
R_{Q A}(a, b)>p\left[\frac{1}{3} C(a, b)+\frac{2}{3} A(a, b)\right]+(1-p) C^{1 / 3}(a, b) A^{2 / 3}(a, b)
$$

follows from (3.9)-(3.11) and (3.16) together with the piecewise monotonicity of $F(x)$.

Case $43\left[(1+\sqrt{2})^{\sqrt{2}} / e-\sqrt[3]{2}\right] /(4-3 \sqrt[3]{2})<p<1$. Then (3.11) leads to

$$
F(\sqrt[6]{2})=\sqrt{2} \log (1+\sqrt{2})-\log \left[\frac{4}{3} p+\sqrt[3]{2}(1-p)\right]-1<0
$$

Equations (3.9) and (3.10) together with inequality (3.17) imply that there exists $0<\delta_{1}^{*}<$ 1 such that

$$
R_{Q A}(a, b)<p\left[\frac{1}{3} C(a, b)+\frac{2}{3} A(a, b)\right]+(1-p) C^{1 / 3}(a, b) A^{2 / 3}(a, b)
$$

for all $a>b>0$ with $(a-b) /(a+b) \in\left(1-\delta_{1}^{*}, 1\right)$.

Theorem 3.4 The double inequality

$$
\begin{aligned}
\alpha_{4} & {\left[\frac{1}{6} C(a, b)+\frac{5}{6} A(a, b)\right]+\left(1-\alpha_{4}\right) C^{1 / 6}(a, b) A^{5 / 6}(a, b) } \\
<R_{A Q}(a, b) & <\beta_{4}\left[\frac{1}{6} C(a, b)+\frac{5}{6} A(a, b)\right]+\left(1-\beta_{4}\right) C^{1 / 6}(a, b) A^{5 / 6}(a, b)
\end{aligned}
$$

holds for all $a, b>0$ with $a \neq b$ if and only if $\alpha_{4} \leq 6\left[\sqrt{2} \mathrm{e}^{(\pi / 4-1)}-\sqrt[6]{2}\right] /(7-6 \sqrt[6]{2})=0.4210 \ldots$ and $\beta_{4} \geq 12 / 25$. 
Proof Since $R_{A Q}(a, b), A(a, b)$, and $C(a, b)$ are symmetric and homogenous of degree one, without loss generality, we assume that $a>b>0$. Let $v=(a-b) /(a+b), x=\sqrt[6]{1+v^{2}}$, and $p \in(0,1)$. Then $v \in(0,1), x \in(1, \sqrt[6]{2})$ and (1.1), (1.3), and (1.5) lead to

$$
\begin{aligned}
& \log \frac{R_{A Q}(a, b)}{p\left[\frac{1}{6} C(a, b)+\frac{5}{6} A(a, b)\right]+(1-p) C^{1 / 6}(a, b) A^{5 / 6}(a, b)} \\
& \quad=\log \sqrt{1+v^{2}}+\frac{\arctan (v)}{v}-\log \left[p\left(\frac{1}{6} v^{2}+1\right)+(1-p) \sqrt[6]{1+v^{2}}\right]-1 \\
& \quad=3 \log (x)+\frac{\arctan \left(\sqrt{x^{6}-1}\right)}{\sqrt{x^{6}-1}}-\log \left[p\left(\frac{1}{6} x^{6}+\frac{5}{6}\right)+(1-p) x\right]-1 .
\end{aligned}
$$

Let

$$
G(x)=3 \log (x)+\frac{\arctan \left(\sqrt{x^{6}-1}\right)}{\sqrt{x^{6}-1}}-\log \left[p\left(\frac{1}{6} x^{6}+\frac{5}{6}\right)+(1-p) x\right]-1 .
$$

Then simple computations lead to

$$
\begin{aligned}
& G\left(1^{+}\right)=0, \quad G(\sqrt[6]{2})=\log (\sqrt{2})+\frac{\pi}{4}-\log \left[\frac{7}{6} p+\sqrt[6]{2}(1-p)\right]-1, \\
& G^{\prime}(x)=\frac{3 x^{5}}{\left(x^{6}-1\right)^{3 / 2}} G_{1}(x)
\end{aligned}
$$

where

$$
\begin{aligned}
& G_{1}(x)=\frac{\sqrt{x^{6}-1}\left[-p x^{11}+4(1-p) x^{6}+7 p x^{5}+2(1-p)\right]}{x^{5}\left[p\left(x^{6}+5\right)+6(1-p) x\right]}-\arctan \left(\sqrt{x^{6}-1}\right), \\
& G_{1}(1)=0, \quad G_{1}(\sqrt[6]{2})=\frac{5[\sqrt[6]{2}(1-p)+p]}{6 \sqrt[6]{2}(1-p)+7 p}-\frac{\pi}{4} \\
& G_{1}^{\prime}(x)=-\frac{\left(x^{6}-1\right)^{3 / 2}}{x^{6}\left[p\left(x^{6}+5\right)+6(1-p) x\right]^{2}} g(x),
\end{aligned}
$$

where $g(x)$ is defined as in Lemma 2.6.

We divide the proof into four cases.

Case $1 p=12 / 25$. Then it follows from (3.18)-(3.23) and Lemma 2.6(1) that

$$
R_{A Q}(a, b)<\frac{12}{25}\left[\frac{1}{3} C(a, b)+\frac{2}{3} A(a, b)\right]+\frac{13}{25} C^{1 / 3}(a, b) A^{2 / 3}(a, b) .
$$

Case $20<p<12 / 25$. Let $v>0$ and $v \rightarrow 0^{+}$, then power series expansion leads to

$$
\begin{aligned}
\log \sqrt{1+v^{2}}+\frac{\arctan (v)}{v}-\log \left[p\left(\frac{1}{6} v^{2}+1\right)+(1-p) \sqrt[6]{1+v^{2}}\right]-1 \\
=\left(\frac{1}{30}-\frac{5}{72} p\right) v^{4}+O\left(v^{6}\right)
\end{aligned}
$$


Equations (3.18), (3.19), and (3.24) lead to the conclusion that there exists $0<\delta_{2}<1$ such that

$$
R_{A Q}(a, b)>p\left[\frac{1}{3} C(a, b)+\frac{2}{3} A(a, b)\right]+(1-p) C^{1 / 3}(a, b) A^{2 / 3}(a, b)
$$

for all $a>b>0$ with $(a-b) /(a+b) \in\left(0, \delta_{2}\right)$.

Case $3 p=6\left[\sqrt{2} \mathrm{e}^{(\pi / 4-1)}-\sqrt[6]{2}\right] /(7-6 \sqrt[6]{2})$. Then, from (3.20) and (3.22) together with numerical computations, we get

$$
G(\sqrt[6]{2})=0, \quad G_{1}(\sqrt[6]{2})=-0.0033 \cdots<0
$$

Let $\mu_{0}=1.0577 \ldots$ be the number given in Lemma 2.6(2). Then we divide the discussion into two subcases.

Subcase $1 x \in\left(1, \mu_{0}\right]$. Then $G_{1}(x)>0$ for $x \in\left(1, \mu_{0}\right]$ follows easily from (3.22) and (3.23) together with Lemma 2.6(2).

Subcase $2 x \in\left(\mu_{0}, \sqrt[6]{2}\right)$. Then Lemma 2.6(2) and (3.23) lead to the conclusion that $G_{1}(x)$ is strictly decreasing on the interval $\left[\mu_{0}, \sqrt[6]{2}\right)$. Then, from (3.25) and Subcase 1 , we know that there exists $\mu_{1} \in\left(\mu_{0}, \sqrt[6]{2}\right)$ such that $G_{1}(x)>0$ for $x \in\left[\mu_{0}, \mu_{1}\right)$ and $G_{1}(x)<0$ for $x \in$ $\left(\mu_{1}, \sqrt[6]{2}\right)$.

It follows from Subcases 1 and 2 together with (3.21) that $G(x)$ is strictly increasing on $\left(1, \mu_{1}\right]$ and strictly decreasing on $\left[\mu_{1}, \sqrt[6]{2}\right)$. Therefore,

$$
R_{A Q}(a, b)>p\left[\frac{1}{3} C(a, b)+\frac{2}{3} A(a, b)\right]+(1-p) C^{1 / 3}(a, b) A^{2 / 3}(a, b)
$$

follows from (3.18)-(3.20) and (3.25) together with the piecewise monotonicity of $G(x)$.

Case $46\left[\sqrt{2} \mathrm{e}^{(\pi / 4-1)}-\sqrt[6]{2}\right] /(7-6 \sqrt[6]{2})<p<1$. Then (3.21) leads to

$$
G(\sqrt[6]{2})=\log (\sqrt{2})+\frac{\pi}{4}-\log \left[\frac{7}{6} p+\sqrt[6]{2}(1-p)\right]-1<0 .
$$

Equations (3.18) and (3.19) together with inequality (3.26) imply that there exists $0<$ $\delta_{2}^{*}<1$ such that

$$
R_{A Q}(a, b)<p\left[\frac{1}{3} C(a, b)+\frac{2}{3} A(a, b)\right]+(1-p) C^{1 / 3}(a, b) A^{2 / 3}(a, b)
$$

for all $a>b>0$ with $(a-b) /(a+b) \in\left(1-\delta_{2}^{*}, 1\right)$.

\section{Results and discussion}

In this paper, we provide the optimal upper and lower bounds for the Sándor-Yang means $R_{Q A}(a, b)$ and $R_{A Q}(a, b)$ in terms of combinations of the arithmetic mean $A(a, b)$ and the contra-harmonic mean $C(a, b)$. Our approach may have further applications in the theory of bivariate means.

\section{Conclusion}

In the article, we find several best possible bounds for the Sándor-Yang means $R_{Q A}(a, b)$ and $R_{A Q}(a, b)$. These results are improvements and refinements of the previous results. 


\section{Funding}

The research was supported by the Natural Science Foundation of China (Grants Nos. 61673169, 61374086, 11371125, 11401191), the Tianyuan Special Funds of the National Natural Science Foundation of China (Grant No. 11626101) and the Natural Science Foundation of the Department of Education of Zhejiang Province (Grant No. Y201635325).

\section{Competing interests}

The authors declare that they have no competing interests.

\section{Authors' contributions}

All authors contributed equally to the writing of this paper. All authors read and approved the final manuscript.

\section{Author details}

${ }^{1}$ School of Economics and Management, Wenzhou Broadcast and TV University, Wenzhou, China. ${ }^{2}$ Department of Mathematics, Huzhou University, Huzhou, China. ${ }^{3}$ School of Distance Education, Huzhou Broadcast and TV University, Huzhou, China.

\section{Publisher's Note}

Springer Nature remains neutral with regard to jurisdictional claims in published maps and institutional affiliations.

Received: 4 January 2018 Accepted: 24 May 2018 Published online: 30 May 2018

\section{References}

1. Chu, Y.-M., Wang, M.-K., Qiu, S.-L.: Optimal combinations bounds of root-square and arithmetic means for Toader mean. Proc. Indian Acad. Sci. Math. Sci. 122(1), 41-51 (2012) Available online at https://link.springer.com/article/10.1007\%2Fs12044-012-0062-y

2. Hua, Y., Qi, F.: The best bounds for Toader mean in terms of the centroidal and arithmetic means. Filomat 28(4), 775-780 (2014) Available at http://www.pmf.ni.ac.rs/filomat

3. Jiang, W.-D., Cao, J., Qi, F.: Sharp inequalities for bounding Seiffert mean in terms of the arithmetic, centroidal, and contra-harmonic means. Math. Slovaca 66(5), 1115-1118 (2016) Available at https://doi.org/10.1515/ms-2016-0208

4. Qian, W.-M., Chu, Y.-M.: Sharp bounds for a special quasi-arithmetic mean in terms of arithmetic and geometric means with two parameters. J. Inequal. Appl. 2017, Article ID 274 (2017) Available at https://doi.org/10.1186/s13660-017-1550-5

5. Chu, Y.-M., Zhao, T.-H., Song, Y.-Q.: Sharp bounds for Neuman-Sándor mean in terms of the convex combination of quadratic and first Seiffert means. Acta Math. Sci. 34B(3), 797-806 (2014) Available at https://doi.org/10.1016/S0252-9602(14)60050-3

6. Chu, Y.-M., Hou, S.-W.: Sharp bounds for Seiffert mean in terms of contraharmonic mean. Abstr. Appl. Anal. 2012 Article ID 425175 (2012) Available at https://doi.org/10.1155/2012/425175

7. Chu, Y.-M., Wang, M.-K., Ma, X.-Y.: Sharp bounds for Toader mean in terms of contraharmonic mean with applications. J. Math. Inequal. 7(2), 161-166 (2013) Available online at https://dx.doi.org/10.7153/jmi-07-15

8. Jiang, W.-D., Qi, F.: Sharp bounds for the Neuman-Sándor mean in terms of the power and contraharmonic means. Cogent Math. 2, Article ID 995951 (2015) Available online at https://doi.org/10.1080/23311835.2014.995951

9. Jiang, W.-D., Qi, F.: A double inequality for the combination of Toader mean and the arithmetic mean in terms of the contraharmonic mean. Publ. Inst. Math. 99(113), 237-242 (2016) Available online at http://www.emis.ams.org/journals/PIMB/113/22.html

10. Chu, Y.-M., Long, B.-Y.: Bounds of the Neuman-Sándor mean using power and identric means. Abstr. Appl. Anal. 2013 Article ID 832591 (2013) Available online at https://doi.org/10.1155/2013/832591

11. Qi, F., Li, W.-H.: A unified proof of several inequalities and some new inequalities involving Neuman-Sándor mean. Miskolc Math. Notes 15(2), 665-675 (2014) Available online at http://mat76.mat.uni-miskolc.hu/mnotes/contents/15/2

12. Jiang, W.-D., Qi, F.: Sharp bounds for Neuman-Sándor's mean in terms of the root-mean-square. Period. Math. Hung 69(2), 134-138 (2014) Available online at https://doi.org/10.1007/s10998-014-0057-9

13. Chu, Y.-M., Wang, M.-K., Qiu, S.-L., Qiu, Y.-F.: Sharp generalized Seiffert mean bounds for Toader mean. Abstr. Appl. Anal. 2011, Article ID 605259 (2011) Available online at https://doi.org/10.1155/2011/605259

14. Jiang, W.-D., Qi, F.: Some sharp inequalities involving Seiffert and other means and their concise proofs. Math. Inequal. Appl. 15(4), 1007-1017 (2012) Available online at https://dx.doi.org/10.7153/mia-15-86

15. Neuman, E., Sándor, J.: On the Schwab-Borchardt mean. Math. Pannon. 14(2), 253-266 (2003) Available online at http://mathematica-pannonica.ttk.pte.hu/index_elemei/mp14-2/mp14-2-253-266.pdf

16. Neuman, E., Sándor, J.: On the Schwab-Borchardt mean II. Math. Pannon. 17(1), 49-59 (2006) Available online at http://www.emis.ams.org/journals/MP/index_elemei/mp17-1/mp17-1-049-059.pd

17. Neuman, E.: On a new bivariate mean. Aequ. Math. 88(3), 277-289 (2014) Available online at https://link.springer.com/article/10.1007\%2Fs00010-013-0224-8

18. Lin, T.P.: The power mean and the logarithmic mean. Am. Math. Mon. 81, 879-883 (1974) Available online at https://www.jstor.org/stable/2319447?seq=1\#page_scan_tab_contents

19. Stolarsky, K.B.: The power and generalized logarithmic means. Am. Math. Mon. 87(7), 545-548 (1980) Available online at https://www.jstor.org/stable/2321420?origin=crossref

20. Hästö, P.A.: Optimal inequalities between Seiffert's mean and power means. Math. Inequal. Appl. 7(1), 47-53 (2004) Available online at https://dx.doi.org/10.7153/mia-07-06

21. Chu, Y.-M., Zhang, X.-M., Wang, G.-D.: The Schur geometrical convexity of the extended mean values. J. Convex Anal. 15(4), 707-718 (2008) Available online at http://www.heldermann.de/JCA/JCA15/JCA154/jca15048.htm

22. Chu, Y.-M., Xia, W.-F.: Two optimal double inequalities between power mean and logarithmic mean. Comput. Math. Appl. 60(1), 83-89 (2010) Available online at https://linkinghub.elsevier.com/retrieve/pii/S08981221 10003032 
23. Neuman, E.: Inequalities for the Schwab-Borchardt mean and their applications. J. Math. Inequal. 5(4), 601-609 (2011) Available online at https://dx.doi.org/10.7153/jmi-05-52

24. Chu, Y.-M., Wang, M.-K., Jiang, Y.-P., Qiu, S.-L.: Concavity of the complete elliptic integrals of the second kind with respect to Hölder means. J. Math. Anal. Appl. 395(2), 637-642 (2012) Available online at https://doi.org/10.1016/j.jmaa.2012.05.083

25. Chu Wang, M.-K.: Optimal Lehmer mean bounds for the Toader mean. Results Math. 61(3-4), 223-229 (2012) Available online at https://ink.springer.com/article/10.1007\%2Fs00025-010-0090-9

26. Neuman, E.: A note on a certain bivariate mean. J. Math. Inequal. 6(4), 637-643 (2012) Available online at https://dx.doi.org/10.7153/jmi-06-62

27. Chu, Y.-M., Qiu, Y.-F., Wang, M.-K.: Hölder mean inequalities for the complete elliptic integrals. Integral Transforms Spec. Funct. 23(7), 521-527 (2012) Available online at https://doi.org/10.1080/10652469.2011.609482

28. Costin, I., Toader, G.: Optimal evaluations of some Seiffert-type means by power means. Appl. Math. Comput. 219(9), 4745-4754 (2013) Available online at https://doi.org/10.1016/j.amc.2012.10.091

29. Chu, Y.-M., Yang, Z.-H., Wu, L.-M.: Sharp power mean bounds for Sándor mean. Abstr. Appl. Anal. 2015, Article ID 172867 (2015) Available online at https://doi.org/10.1155/2015/172867

30. Qi, F., Shi, X.-T., Liu, F.-F., Yang, Z.-H.: A double inequality for an integral mean in terms of the exponential and logarithmic means. Period. Math. Hung. 75(2), 180-189 (2017) Available online at https://link.springer.com/article/10.1007\%2Fs10998-016-0181-9

31. Wang, M.-K., Chu, Y.-M.: Refinements of transformation inequalities for zero-balanced hypergeometric functions. Acta Math. Sci. 37B(3), 607-622 (2017) Available online at https://doi.org/10.1016/S0252-9602(17)30026-7

32. Yang, Z.-H., Chu, Y.-M.: A monotonicity property involving the generalized elliptic integral of the first kind. Math. Inequal. Appl. 20(3), 729-735 (2017) Available online at https://dx.doi.org/10.7153/mia-20-46

33. Yang, Z.-H., Zhang, W., Chu, Y.-M.: Sharp Gautschi inequality for parameter $0<p<1$ with applications. Math. Inequal. Appl. 20(4), 1107-1120 (2017) Available online at https://dx.doi.org/10.7153/mia-2017-20-71

34. Qi, F., Guo, B.-N.: Lévy-Khintchine representation of Toader-Qi mean. Math. Inequal. Appl. 21(2), 421-431 (2018) https://dx.doi.org/10.7153/mia-2018-21-29

35. Yang, Z.-H., Qian, W.-M., Chu, Y.-M., Zhang, W.: On approximating the error function. Math. Inequal. Appl. 21(2), 469-479 (2018) Available online at https://dx.doi.org/10.7153/mia-2018-21-32

36. Wang, M.-K., Chu, Y.-M.: Landen inequalities for a class of hypergeometric functions with applications. Math. Inequal. Appl. 21(2), 521-537 (2018) Available online at https://dx.doi.org/10.7153/mia-2018-21-38

37. Yang, Z.-H., Qian, W.-M., Chu, Y.-M., Zhang, W.: On approximating the arithmetic-geometric mean and complete elliptic integral of the first kind. J. Math. Anal. Appl. 462(2), 1714-1726 (2018) Available online at https://doi.org/10.1016/j.jmaa.2018.03.005

38. Wang, M.-K., Li, Y.-M., Chu, Y.-M.: Inequalities and infinite product formula for Ramanujan generalized modular equation function. Ramanujan J. 46(1), 189-200 (2018) Available online at https://link.springer.com/article/10.1007\%2Fs11139-017-9888-3

39. Yang, Z.-H.: Three families of two-parameter means constructed by trigonometric functions. J. Inequal. Appl. 2013, Article ID 541 (2013) https://link.springer.com/article/10.1186/1029-242X-2013-541

40. Yang, Z.-H., Jiang, Y.-L., Song, Y.-Q., Chu, Y.-M.: Sharp inequalities for trigonometric functions. Abstr. Appl. Anal. 2014 Article ID 601839 (2014) Available online at https://doi.org/10.1155/2014/601839

41. Yang, Z.-H., Chu, Y.-M.: Optimal evaluations for the Sándor-Yang mean by power mean. Math. Inequal. Appl. 19(3), 1031-1038 (2016) Available online at https://dx.doi.org/10.7153/mia-19-76

42. Yang, Y.-Y., Qian, W.-M.: Two optimal inequalities related to the Sândor-Yang mean and one-parameter mean. Commun. Math. Res. 32(4), 352-358 (2016) Available online at http://www.cnki.net/kcms/doi/10.13447/j.1674-5647.2016.04.07.html

43. Neuman, E.: On a new family of bivariate means. J. Math. Inequal. 11(3), 673-681 (2017) Available online at https://doi.org/10.7153/jmi-2017-11-53

44. Xu, H.-Z:: Sharp bounds for Sándor-Yang means in terms of some bivariate means. J. East China Norm. Univ. Natur. Sci. Ed. 2017(4), 41-51 (2017) Available online at http://xblk.ecnu.edu.cn/CN/volumn/volumn_1157.shtml

45. Anderson, G.D., Vamanamurthy, M.K., Vuorinen, M.: Conformal Invariants, Inequalities, and Quasiconformal Maps. Wiley, New York (1997)

46. Biernacki, M., Krzyż, J.: On the monotonicity of certain functionals in the theory of analytic functions. Ann. Univ. Mariae Curie-Skłodowska, Sect. A 9, 135-147 (1955) Available online at https://cms.math.ca/10.4153/CMB-1967-074-9

\section{Submit your manuscript to a SpringerOpen ${ }^{\circ}$ journal and benefit from:}

- Convenient online submission

Rigorous peer review

- Open access: articles freely available online

- High visibility within the field

Retaining the copyright to your article

Submit your next manuscript at $\gg$ springeropen.com 